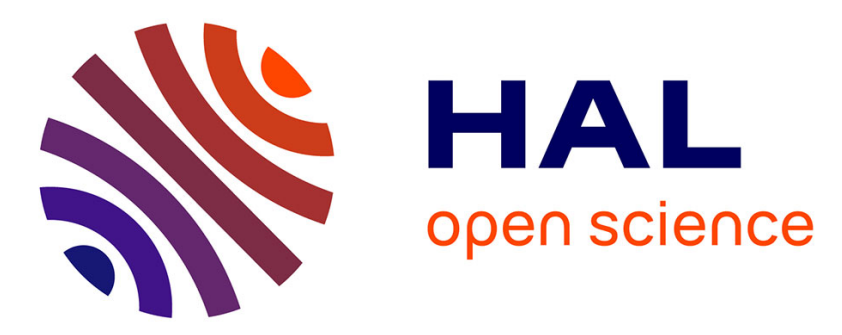

\title{
Stochastic model for curvilinear structure reconstruction using morphological profiles
}

\author{
Seong-Gyun Jeong, Yuliya Tarabalka, Josiane Zerubia
}

\section{To cite this version:}

Seong-Gyun Jeong, Yuliya Tarabalka, Josiane Zerubia. Stochastic model for curvilinear structure reconstruction using morphological profiles. ICIP 2015 - IEEE International Conference on Image Processing, IEEE, Sep 2015, Quebec City, Canada. hal-01152932

\section{HAL Id: hal-01152932 \\ https://inria.hal.science/hal-01152932}

Submitted on 3 Jun 2015

HAL is a multi-disciplinary open access archive for the deposit and dissemination of scientific research documents, whether they are published or not. The documents may come from teaching and research institutions in France or abroad, or from public or private research centers.
L'archive ouverte pluridisciplinaire $\mathbf{H A L}$, est destinée au dépôt et à la diffusion de documents scientifiques de niveau recherche, publiés ou non, émanant des établissements d'enseignement et de recherche français ou étrangers, des laboratoires publics ou privés. 


\title{
STOCHASTIC MODEL FOR CURVILINEAR STRUCTURE RECONSTRUCTION USING MORPHOLOGICAL PROFILES
}

\author{
Seong-Gyun JEONG, Yuliya TARABALKA, and Josiane ZERUBIA \\ INRIA, AYIN research team, 2004 route des Lucioles, 06902 Sophia Antipolis, France \\ E-mails: \{firstname.lastname\} @inria.fr
}

\begin{abstract}
In this work, we propose a stochastic model for curvilinear structure reconstruction using morphological profiles of path opening operator. We apply the support vector machine classifier to obtain initial probabilities to belong to line network for each pixel. Then, we formulate a stochastic optimization problem that detects line segments corresponding to the latent curvilinear structure in a scene. Experimental results on DNA filament and remote sensing images validate the effectiveness of the proposed algorithm when compared to other recent methods.
\end{abstract}

Index Terms - curvilinear structure modeling, morphological profiles, object detection, point process

\section{INTRODUCTION}

Understanding of the latent curvilinear structure has been a challenging problem in computer vision. In biomedical imaging, line detection algorithms have been proposed to analyze complex linear shape of DNA filaments [1] and to diagnose vascular diseases [2]. Road extraction algorithms to interpret geographical information have been studied for remote sensing applications [3].

Template matching algorithms compute linearity scores of the pixels by the inner-product between given image data and predefined line shapes. For example, steerable filters of elongated Gaussian kernel efficiently highlight rotated gradient magnitudes which correspond to curvilinear structure [1, 4]. Morphological filtering [3,5] is popularly employed to detect hidden linear structure with simple shape prior, i.e., adjacency graph to define geography. Türetken et al. [6] formulated an optimization problem with integer programming to connect scattered control points belonging to line networks.

While the automation of the cuvilinear structure extraction tasks has become a critical issue due to the rapid growth of imaging systems, the above mentioned algorithms have following drawbacks: 1) A threshold value is needed to finalize binary classification of the curvilinear structure in both template matching and morphological operator; and 2) Choosing initial conditions and parameter learning are time consuming if an algorithm seeks for a high dimensional solution space.
In [7], stochastic inference of the curvilinear structure has been studied by point process with shape prior constraints. The authors assumed that the entire line network can be disjoint into small line segments. Although this assumption allows to apply the algorithm in various types of dataset, in some cases, straight line segments are inefficient to represent intricate line networks. We extend the probabilistic model of the curvilinear structure [7] by employing a rich curvilinear feature descriptor. We perform a morphological operation [8] to analyze the local structure. It is inspired by Valero et al.'s work [3]. While Valero et al. proposed dynamic thresholding scheme based on the statistics of morphological profiles, we determine the criteria of being curvilinear structure using a supervised learning algorithm. In addition, the line network is reconstructed as an unordered set of line segments to provide vectorized information instead of pixelwise classification.

We want to develop a curvilinear feature descriptor and a fully automatic curvilinear structure extraction algorithm. For this purpose, we construct the feature descriptor which consists of pixel intensity and morphological filtering responses with different length parameters [3]. We also study the optimal dimensionality of the feature vector and the minimum length parameter for the morphological profiles. A support vector machine (SVM) classifier [9] is applied to obtain pixelwise posterior probabilities to belong to the line network. Finally, we minimize the energy on configuration space of line segments to reconstruct salient curvilinear structure in the scene. We validate the proposed algorithm by comparing its performances on DNA filament and remote sensing images with the state-of-the-art approaches.

The rest of this paper is organized as follows: Section 2 reviews path opening operator and morphological profiles. Section 3 proposes the stochastic curvilinear structure modeling with the proposed feature descriptor. Section 4 provides experimental results. Finally, we conclude in Section 5.

\section{CURVILINEAR FEATURE DESCRIPTOR WITH MORPHOLOGICAL PROFILES}

This section reviews the preliminary techniques of path opening operator [8] and morphological profiles [3, 10] to describe curvilinear features, on which the proposed algorithm is based. 

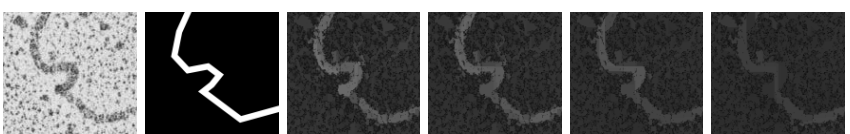

(a) DNA

(b) Truth

(c) $L=50$
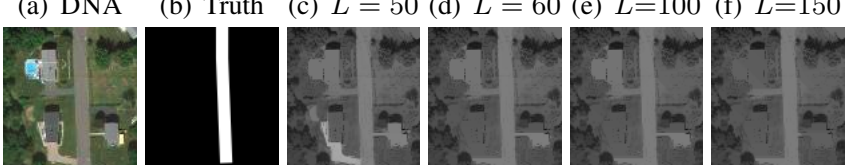

(g) ROAD

(h) Truth

(i) $L=50$ (j) $L=100$

(k) $L=130$ (l) $L=150$

Fig. 1. Left to right: Input image, ground truth, and path opening [8] results with different length parameter $L$. First and second rows correspond to results on DNA and ROAD, respectively. The path opening operator removes small bright objects which are not belonging to a valid path.

\subsection{Path opening}

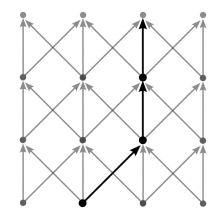

Given a binary image $\mathcal{B} \in \mathbb{R}^{2}$, a morphological operator seeks for similar structural elements on the adjacency graph (figure on the left shows an example of the adjacency graph and a valid path on the graph). Let $\delta(u)=\{v \in \mathcal{B}, u \mapsto v\}$ be the structuring function which defines a path for a given length $L$, where $u \mapsto v$ denotes a directed edge going from node $u$ to $v$. Then, a valid $\delta$-path of length $L$ is represented as $L$-tuple $\mathbf{u}=\left(u_{1}, \ldots, u_{L}\right)$ that satisfies $u_{k+1} \in \delta\left(u_{k}\right)$ for $k=1, \ldots, L-1$. The path opening operator is defined as the union of all valid $\delta$-paths of $L$ in the image.

To generalize the binary path opening operator to grey level image, we decompose in input grey level image into binary images with decreasing order of threshold values. For each decomposed binary image, the morphological operator looks for the longest path $\delta_{\max }$ for all pixels. Finally, the threshold value which corresponds to infimum of the longest path $\delta_{\max }$ among decomposed binary images is assigned as the outcome of the morphological filtering.

\subsection{Curvilinear feature descriptor}

The path opening operator highlights structural similarity of pixels according to the adjacency graph that encodes a curvilinear shape prior. On the other hand, the operator removes bright pixels if such pixels belong to a path shorter than the length parameter $\left(\delta_{\max }<L\right)$. Let $I(i)$ be an intensity value of the pixel $i$ and $M(i ; L)$ be the path opening result at $i$ with length parameter $L$. In this paper, we employ a feature descriptor $\mathbf{f}_{i}$ which combines the pixel intensity and the morphological profiles with increasing order of length parameter $L$. That is

$$
\mathbf{f}_{i}=\left[I(i), M\left(i ; L_{1}\right), \ldots, M\left(i ; L_{\#}\right)\right]^{\top} .
$$

We assume that pixels belonging to the curvilinear structure are brighter than their neighborhoods. Otherwise, we invert

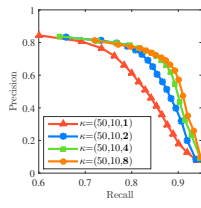

(a) $\mathrm{DNA}(\#)$

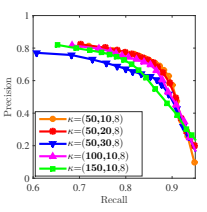

(b) $\operatorname{DNA}\left(L^{*}, \Delta\right)$

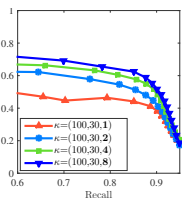

(c) $\operatorname{ROAD}(\#)$

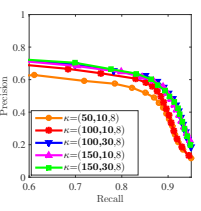

(d) $\operatorname{RoAD}\left(L^{*}, \Delta\right)$
Fig. 2. Precision-and-recall curves for two datasets (DNA and ROAD) by controlling the dimensionality of the feature descriptor ((a) and (c)) and the minimum length parameters $L^{*}$ and increment values $\Delta((\mathrm{b})$ and (d)).

the intensity level of the input image before applying the morphological operator. Comparing to [3], our feature vector needs half size of their morphological profiles; thus, it yields faster computation.

Fig. 1 compares path opening results with different length parameters. With a larger value of the length parameter $L$, we can retrieve longer chain of line structure and remove unexpected noise. If $L$ is too large, the curvilinear structure is considered as a noisy component and then it begins to disappear ( $L=150$ for DNA image). Therefore, a suitable length parameter should be taken into account when we build an efficient feature descriptor. We observe the effect of the parameter $\kappa=\left(L^{*}, \Delta, \#\right)$ on line detection performance (precisionand-recall curve) for all test images, where $L^{*}, \Delta$, and \# denote the minimum length, increment, and the number of morphological operations, respectively. Fig. 2 (a) and (c) compare the performances by increasing the dimensionality of the feature descriptor, for DNA and ROAD datasets, respectively. Fig. 2 (b) and (d) show the effects of $L^{*}$ and $\Delta$. We see that the best performance is reached when the feature vector includes promising path opening results for a given dataset. We apply path opening operator on the DNA images with $\kappa_{1}=(50,10,8)$ and ROAD images with $\kappa_{2}=(100,30,8)$, respectively. Please recall Fig. 1 to see the correspondence between our choice of $\kappa$ and visual results. Also, the values of the feature descriptor are normalized to a range of $[0,1]$.

\section{STOCHASTIC IMAGE MODEL FOR CURVILINEAR STRUCTURE RECONSTRUCTION}

\subsection{Support Vector Machines for initial estimation}

We employ SVM [9] for an initial classification of the linear structure. Let $\mathcal{D}=\left\{\left(\mathbf{f}_{i}, y_{i}\right)\right\}_{i=1}^{K}$ be a training set, where $\mathbf{f}_{i}$ denotes our feature descriptor and $y_{i} \in\{-1,1\}$ is a corresponding label of the data point. In practice, we randomly select 50 training data points for positive and negative samples, respectively. To obtain a linear hyperplane, we solve following quadratic programming problem:

$$
\begin{aligned}
\min _{b, \mathbf{w}, \boldsymbol{\eta}} & \frac{1}{2}\|\mathbf{w}\|_{2}^{2}+C \sum_{i=1}^{K} \eta_{i} \\
\text { s.t. } & y_{i}\left(\mathbf{w}^{\top} \mathbf{f}_{i}+b\right) \geq 1-\eta_{i}, \boldsymbol{\eta} \succeq 0, i=1, \ldots, K
\end{aligned}
$$




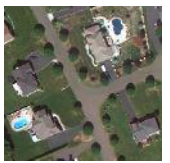

(a) Input

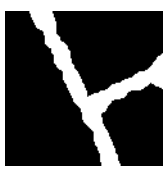

(b) Truth

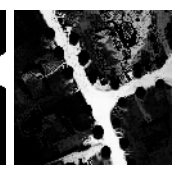

(c) $\operatorname{SVM}\left(p_{i}\right)$

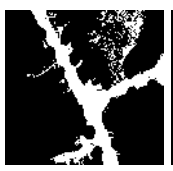

d) $\operatorname{SVM}\left(y_{i}\right)$

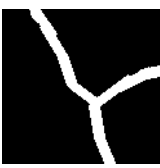

(e) Proposed
Fig. 3. For the input image (a), we compare the manually labeled result (b), outcome of the SVM classifier as the posterior probabilities (c) and binary classification (d). The proposed stochastic model (e) refines the weak points of the SVM by sampling line segments with geometry prior constraints.

where $\mathbf{w}$ is support vector, $K$ is the total number of training data, $b$ represents the bias of the hyperplane, and $\eta$ denotes slack variables for regression. $C$ controls the importance of such regression term.

The distance of the data point from the hyperplane can be considered as a probability to belong to curvilinear structure. In [11], a posterior probability is approximated by a sigmoid function:

$$
p_{i}=\operatorname{Pr}\left(y_{i}=1 \mid \mathbf{f}_{i}\right)=\frac{1}{1+\exp \left(\alpha g_{i}+\beta\right)},
$$

where $g_{i}=\mathbf{w}^{\boldsymbol{\top}} \mathbf{f}_{i}+b$ is the decision function of the SVM classifier, and the label $y_{i}=\operatorname{sign}\left(g_{i}\right)$. The parameters for regularization $(\alpha$ and $\beta$ ) are estimated by maximum likelihood.

\subsection{Stochastic modeling of curvilinear structure}

The outcomes of the SVM show holes and jitters due to the misclassification. The linear SVM fails to classify the curvilinear structure if the training dataset is badly chosen or test data points lie within the margin (see Fig. 3).

To make up for such drawbacks, we propose a stochastic model which employs the curvilinear feature of morphological profiles (Sec. 2.2) and the simple spatial interaction term. We recursively sample short line segments corresponding to the latent line network, where each line segment is expressed in a tuple of image coordinate, length, and orientation: $\ell_{i}=\left(i, l_{i}, \theta_{i}\right)$. Then, we design the data likelihood energy to make line objects agree with the image data, and the shape prior constraints to prevent the congestion of line segments. The data likelihood is defined as:

$$
E_{\text {data }}\left(\ell_{i}\right)=\sum_{j \in \ell_{i}}\left\|\mathbf{f}_{j}-\overline{\mathbf{f}}\right\|_{2}^{2}-\lambda \sum_{j \in \ell_{i}} p_{j},
$$

where $\overline{\mathbf{f}}=\frac{\sum_{k, y_{k}=1} p_{k} \mathbf{f}_{k}}{\sum_{k, y_{k}=1} p_{k}}$ is the centroid of the positive labeled data points, and $\lambda$ controls the relative importance of two terms. More specifically, the first term measures the similarity between the SVM classification results and feature vectors belonging to the line segment $\ell_{i}$. The second term regulates the sampling space depending on the initial estimation.

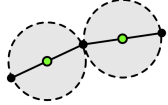

(a)

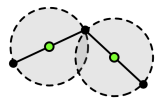

(b)

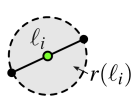

(c)

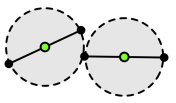

(d)

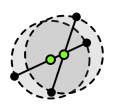

(e)
Fig. 4. Spatial interactions of the line segments are illustrated in the increasing order of $E_{\text {prior. }}$. Our system prefers to choose a smooth curvature and connected line configuration.

We propose the shape prior constraints based on intersection and union of the augmented line segments $r(\ell)$, and the end-to-end distance $d\left(\ell_{m}, \ell_{n}\right)$, given by

$E_{\text {prior }}\left(\ell_{m}, \ell_{n}\right)= \begin{cases}d\left(\ell_{m}, \ell_{n}\right)+\frac{\left|r\left(\ell_{m}\right) \cap r\left(\ell_{n}\right)\right|}{\left|r\left(\ell_{m}\right) \cup r\left(\ell_{n}\right)\right|} & \text { if } \exists \ell_{m} \sim \ell_{n}, \\ \xi & \text { otherwise, }\end{cases}$

where $|r(\cdot)|$ counts the number of pixels falling in the area. $\ell_{m} \sim \ell_{n}$ denotes symmetric relation between two line segments if their center distance is closer than $\frac{l_{m}+l_{n}}{2}$. A singular line segment will be penalized by a constant value $\xi$ for the absence of neighborhood. Fig. 4 shows examples of spatial interactions of line segments.

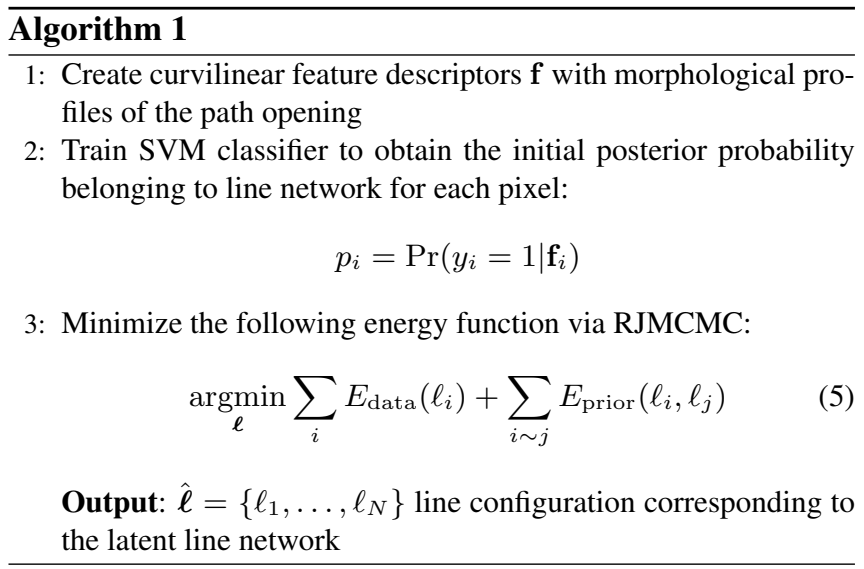

Algorithm 1 summarizes the procedure to reconstruct line network using the proposed stochastic curvilinear structure model. We minimize the given energy function (5) over the configuration space via Reversible jump Markov chain Monte Carlo (RJMCMC) sampler [12, 13]. We refer the reader to [7] for implementation details of the RJMCMC optimization.

\section{EXPERIMENTAL RESULTS}

We test the proposed algorithm on DNA filament images ${ }^{1}$ and road networks in remote sensing images ${ }^{2}$. To evaluate its performances, we compare the proposed method with Valero et al.'s algorithm [3], which proposes morphological profiles to

\footnotetext{
${ }^{1}$ https://www.biochem.wisc.edu/faculty/inman/empics/dna-prot.htm

${ }^{2} \mathrm{http} / / /$ cvlab.epfl.ch/data/delin
} 
Table 1. Quantitative evaluation of Valero et al.'s algorithm [3], Jeong et al.'s algorithm [7], and the proposed algorithm. Boldfaced numbers denote the best performance in each test.

\begin{tabular}{|c|c|c|c|c|c|c|c|c|c|}
\hline & \multicolumn{3}{|c|}{ Recall } & \multicolumn{3}{|c|}{ Precision } & \multicolumn{3}{|c|}{ Accuracy } \\
\hline & [3] & [7] & Proposed & [3] & [7] & Proposed & [3] & [7] & Proposed \\
\hline DNA1 & 49.11 & 62.22 & 71.17 & 88.08 & 67.55 & 73.00 & 97.14 & 97.96 & 98.43 \\
\hline DNA2 & 63.47 & 73.81 & 77.47 & 85.12 & 84.78 & 84.22 & 97.53 & 98.49 & 98.23 \\
\hline DNA3 & 56.42 & 66.13 & 71.29 & 89.94 & 57.83 & 72.25 & 97.36 & 97.62 & 98.12 \\
\hline ROAD 1 & 61.29 & - & 88.52 & 90.42 & - & 88.13 & 94.62 & - & 98.12 \\
\hline ROAD2 & 43.50 & - & 81.81 & 88.87 & - & 85.13 & 90.97 & - & 97.59 \\
\hline ROAD3 & 48.69 & - & 73.49 & 94.99 & - & 85.70 & 93.20 & - & 97.07 \\
\hline
\end{tabular}

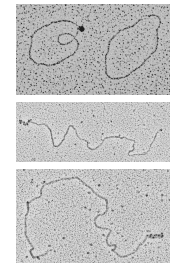

(a) Input

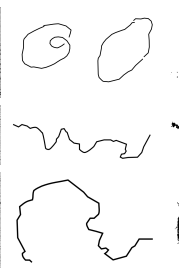

(b) Truth
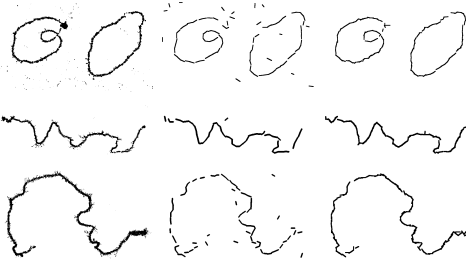

(c) [3]

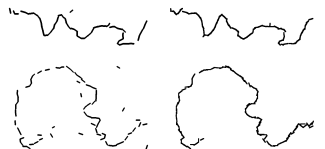

(d) [7]

(e) Proposed

Fig. 5. Visual comparisons of the proposed algorithm with manually labeled ground truth, Valero et al.'s algorithm [3], and Jeong et al.'s algorithm [7] for DNA filament images. Top to bottom: DNA1, DNA2, and DNA3.

detect road networks, and Jeong et al.'s model [7], which employs rotatable gradient information as data term.

In this work, we use LibSVM [14] to solve eq. (1), and $C$ is fixed to 0.1 to control the relative importance between two terms of eq. (1). For parameters of the energy function, $\lambda$ is set to 255 and $\xi$ is upon the dataset type: 2500 for remote sensing and 250 for DNA filament images. The area of the augmented line segment is defined as $r\left(\ell_{i}\right)=\{x:\|x-i\|<$ $\left.\frac{l_{i}}{2}\right\}$ (see Fig. 4 (c)).

For visual comparisons, Fig. 5 and Fig. 6 show the line detection results of DNA filament and road network, respectively. Jeong et al.'s algorithm [7] cannot detect road network, where the gradient magnitude inside of the road is flat. It means that the gradient information is insufficient to describe curvilinear structure in the case of remote sensing applications. In addition, due to the fussy prior constraints, the outcome of [7] makes discontinuities on the line. The dynamic thresholding algorithm using morphological profiles shows misclassification around impurities, e.g., tree, car, and noise. The proposed algorithm well visualizes structural information without redundancies.

We also provide the quantitative evaluation with the following measurements:

$$
\begin{gathered}
\text { Recall }=\frac{T P}{T P+F N}, \\
\text { Precision }=\frac{T P}{T P+F P}, \\
\text { Accuracy }=\frac{T P+T N}{T P+T N+F P+F N},
\end{gathered}
$$

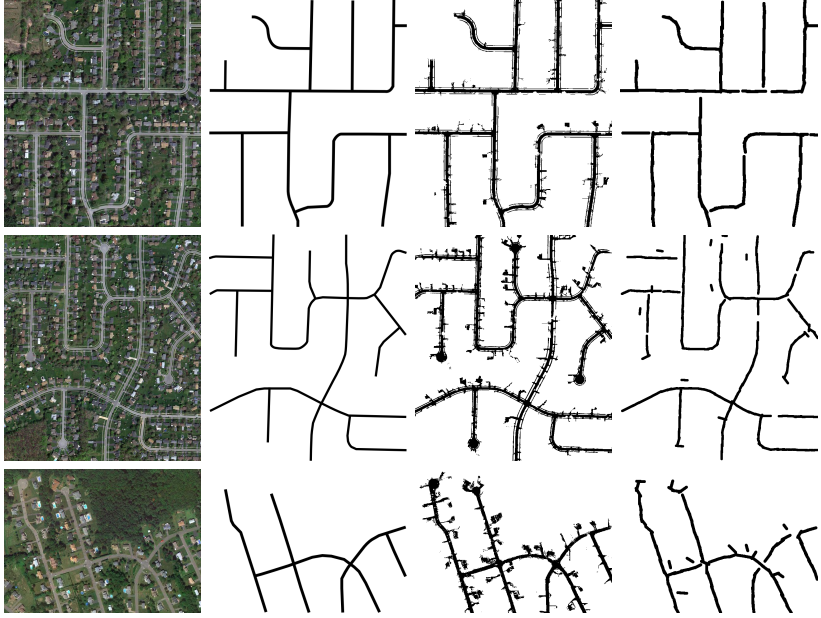

(a) Input

(b) Truth

(c) [3]

(d) Proposed

Fig. 6. Visual comparisons of the proposed algorithm with manually labeled ground truth, and Valero et al.'s algorithm [3] for remote sensing images. Top to bottom: ROAD1, ROAD2, and ROAD3.

where $T P, T N, F P$, and $F N$ denote the true positive, true negative, false positive, and false negative rates, respectively. Table 1 summarizes the quantitative results. The proposed algorithm shows the highest recall and accuracy performances in general.

\section{CONCLUSIONS}

We proposed a new curvilinear structure reconstruction algorithm which exploits morphological profiles of path opening operator. We studied the parameters of the curvilinear feature descriptor to find the optimal dimensionality for our system. We performed a weakly supervised method to estimate the posterior probabilities of being a line network for each pixel. To correct the uncertainty of pixelwise classification, we formulated an energy optimization problem that maximizes inner class similarities with the simple geometric shape constraints. We demonstrated experimental results on DNA filament and road network images to compare performances of proposed algorithm with other methods. 


\section{REFERENCES}

[1] M. Jacob and M. Unser, "Design of steerable filters for feature detection using Canny-like criteria," IEEE TPAMI, vol. 26, no. 8, pp. 1007-1019, Aug. 2004.

[2] J. J. Staal, M. D. Abramoff, M. Niemeijer, M. A. Viergever, and B. van Ginneken, "Ridge based vessel segmentation in color images of the retina," IEEE TMI, vol. 23, no. 4, pp. 501-509, Apr. 2004.

[3] S. Valero, J. Chanussot, J.A. Bendiktsson, H. Talbot, and B. Waske, "Advanced directional mathematical morphology for the detection of the road network in very high resolution remote sensing images," Pattern Recognition Lett., vol. 31, no. 10, pp. 1120-1127, July 2010.

[4] C. Becker, R. Rigamonti, V. Lepetit, and P. Fua, "Supervised feature learning for curvilinear structure segmentation," in MICCAI, Sept. 2013, pp. 526-533.

[5] P. Soille and H. Talbot, "Directional morphological filtering," IEEE TPAMI, vol. 23, no. 11, pp. 1313-1329, Nov. 2001.

[6] E. Türetken, F. Benmansour, B. Andres, H. Pfister, and P. Fua, "Reconstructing loopy curvilinear structures using integer programming," in Proc. IEEE CVPR, June 2013, pp. 1822-1829.

[7] S.-G. Jeong, Y. Tarabalka, and J. Zerubia, "Marked point process model for curvilinear structures extraction," in EMMCVPR, Jan. 2015, pp. 436-449.

[8] H. Talbot and B. Appleton, "Efficient complete and incomplete path openings and closings," Image and Vision Computing, vol. 25, no. 4, pp. 416-425, Apr. 2007.

[9] V. Vapnik, Statistical learning theory, John Wiley \& Sons, Inc., 1998.

[10] M. Pesaresi and J.A. Benediktsson, "A new approach for the morphological segmentation of high-resolution satellite imagery," IEEE TGR, vol. 39, no. 2, pp. 309320, Aug. 2001.

[11] J. Platt, "Probabilistic outputs for support vector machines and comparison to regularized likelihood methods," in Advances in large margin classifiers, A. Smola, P. Bartlett, B. Schölkopf, and D. Schuurmans, Eds. MIT Press, Cambridge, MA, 2000.

[12] C. J. Geyer and J. Møller, "Simulation procedures and likelihood inference for spatial point processes," Scand. J. Stat, vol. 21, pp. 359-373, 1994.

[13] P. J. Green and A. Mira, "Delayed rejection in reversible jump Metropolis-Hastings," Biometrika, vol. 88, no. 4, pp. 1035-1053, 2001.
[14] C.-C. Chang and C.-J. Lin, "LIBSVM: A library for support vector machines," ACM Transactions on Intelligent Systems and Technology, vol. 2, no. 3, pp. 27:1-27:27, 2011. 